\title{
GALAXY MORPHOLOGY, INITIAL CONDITIONS AND THE HUBBLE SEQUENCE
}

\author{
P.R. WILLIAMS AND A.H. NELSON \\ Department of Physics and Astronomy, University of Wales \\ College Cardiff, PO Box 913, Cardiff, CF2 3YB
}

We have carried out over 120 galaxy formation N-body simulations modeling gravity, gas dynamics and star formation using TREESPH on a parallel computer. Our aims were to investigate whether or not numerical galaxies formed from idealized cosmological perturbations can account for the Hubble sequence and the diversity of disk galaxies observed in the field.

The initial conditions used were isolated uniform density spheres in solid body rotation and in Hubble expansion. One tenth of the total mass was initially in gaseous form and nine tenths in dark matter. Perturbations were further constrained to be bound, and have a dimensionless spin parameter $\lambda$, (a measure of rotational support,) $0.03 \leq \lambda \leq 0.11$.

Our parameter survey has shown that:

- The range of disk sizes, bulge sizes, bulge-to-disk ratios and arm definition observed in field galaxies is comparable to that found in the numerical galaxies.

- There is a well defined link between the physical parameters of the initial perturbation and the parameters defining the final galaxy morphology. But there is no direct one-to-one mapping from the parameters of the initial perturbation to Hubble type.

- Perturbations which decoupled from the Hubble flow at $z>10$ formed galaxies which were found to be too compact, rotated too quickly and converted most of their mass into stars at redshifts $>5$. Madau et al. (1996) have found that the global star formation rate of the Universe rises from $z \simeq 5$ to a peak at $z \simeq 1$ and then decays. Therefore a cosmology in which most galaxies form at late times is preferred.

\section{References}

Madau, P., Ferguson, H.C., Dickinson, M.E., Giavalisco, M., Steidel, C.C, Fruchter, A. 1996, MNRAS, 283, 1388 